computers and microcomputers. The 79 papers describe programs and systems which range from the grandiose to the trivial. Several of the papers describe large menu-driven systems for processing sequences, including printing out sequences in various formats, translating them into amino acid sequences and searching for restriction sites to mention but a few of the options. Other contributions are devoted to more specialized tasks, such as working out restriction maps, ascertaining the homology between sequences, determining the most stable base-pairing scheme in an RNA chain, calculating the molecular size of fragments observed in polyacrylamide gels and even designing your experiment for you. There is only one paper devoted to the graphic display of three-dimensional structures; this is notable for being implemented on a microcomputer.

Molecular biologists engaged in producing nucleic acid sequences or cogitating on their significance are likely to find something of interest in the two books. It may be that the author will send them a listing when asked (some offer so to do, others are silent on this point), that the program is written in a language and dialect familiar to their local computer, and that appropriate hardware is available. If all this is so, such researchers may be saved hours of work reinventing the wheel. But it is unclear whether the programs themselves have been verified in any way, and hard to tell how robust they are against misuse or how easy they are to use.

Most of the programs concerned with homology and structure prediction are based on the dynamic programming method discovered, apparently simultaneously, by several workers in the 1970s. This method, based on an argument by induction for ascertaining the minimum number of insertions, deletions or changes required to transform one sequence into another, is well described in Time Warps, String Edits and Macromolecules.

In this book, various contributors explain how almost identical problems arise in several disparate fields, including speech recognition. Here the pressure wave corresponding to the utterance of a word is sampled at discrete times and a resulting vector compared with that of a standard word. Since people vary in the rate at which they speak, the sampling rate must be varied in some way; that is, time must be warped.

Much of Sankoff and Kruskal's volume should be accessible to molecular biologists interested in the intricacies of sequence comparisons and similar problems, and should serve a useful role in acquainting them with the state of play in this area. Other parts are more mathematical and thus more suitable for the computer specialist.

E.G. Richards is Senior Lecturer in Biophysics at King's College, University of London.

\section{How to crunch your numbers}

\author{
Gordon C.K. Roberts
}

Biodata Handling with Microcomputers. By R.B. Barlow.

Elsevier Biosoft, 68 Hills Road,

Cambridge CB2 ILA, UK: 1983. Pp.261.

£18. \$30. Disks £30. \$50.

EVEN the least expensive "home computers" are capable of carrying out all the statistical analysis that most biologists will require - and usually before the answer can be obtained from the computer centre. The main obstacle to practical realization of this is, of course, the limited availability of appropriate programs. Behind the somewhat mysterious title (what is "biodata"?), this book goes a considerable way towards meeting the need. It contains some 50 programs which cover most simple statistical procedures, from standard deviations and significance tests through analysis of variance to linear and non-linear (iterative) regression.

The book contains listings of the programs (in Commodore PET BASIC), repro-

\section{Pascal in practice}

\section{Graham H. Kirby}

Pascal Applications for the Sciences:

A Self-Teaching Guide.

By Richard E. Crandall.

Wiley: 1984. Pp.246. Pbk £13.90,

$\$ 16.95$.

THE number of textbooks which deal with computer programming in Pascal is very large and still increasing. So it is refreshing to find that Richard Crandall's book concentrates on applying the language. The author's aim was to provide a text which would enable students of mathematics, physics, chemistry and biology to learn scientific programming in Pascal. Applications in each of these specific subjects follow a general introduction to simple numerical programming.

The book is a self-teaching guide, consisting of text, exercises, answers, more text and so on. Some exercises involve modification of the many programs presented in the text whilst others are distinctly ambitious. Many answers are short comments and hints rather than code. One of the good features is the emphasis on graphics to display computed results, rather than on the printing of tables of

- Reston/Prentice-Hall have just published Scientific Pascal by Harley Flanders, a book written specifically to teach practical programming to scientists and students of science. Price is hbk $\$ 29.95, £ 28.45 ;$ pbk $\$ 21.95, £ 20.85$. duced from a dot matrix printer, while the text is reproduced from typescript - both are entirely legible, although the multistatement lines and lack of indenting make the programs harder to follow than they need be. White space is prominent, since many pages are under two-thirds full, and there are several rather un-funny cartoons.

Each program is accompanied by a brief but generally adequate commentary describing what is going on, and in many cases also by an even briefer outline of the underlying mathematics. Beginners may need a statistical text to amplify some of the explanations if, as the author hopes, they wish not only to use this book as a "toolkit" but also to understand how the tools work. In addition each program has a sample output, the examples being drawn primarily from pharmacology.

The programs cover clearly the most widely used statistical procedures, and are available on disk for Commodore PET, Apple and Commodore 64 computers. The combination of disk and book certainly adds up to a useful elementary statistical package.

Gordon C. K. Roberts is in the Division of Physical Biochemistry at the National Institute for Medical Research, London.

numbers, and a microcomputer with graphics facilities and Pascal would provide the ideal basis from which to benefit from the book. Also commendable is the provision of source code for libraries of Pascal procedures covering graphics, matrix handling, statistics and specialized mathematical functions. Of course, the graphics library is hardware specific (for Tektronix 4012 terminals).

A book such as this, modestly-priced and devoted to applications, has insufficient space to introduce the language itself. Readers will require either a prior knowledge or an introductory textbook on Pascal programming. Without one or the other, the benefits of Pascal will not be appreciated since Crandall has concentrated entirely on the mathematical modelling type of application using simple numerical and statistical techniques. This is a pity because the full power of Pascal is not demonstrated - I noted very few occurrences of the type char, only one of records (for complex numbers) and of the case statement, and none of pointer types. The programs could be re-written in FORTRAN or BASIC with few changes.

Science students will find this book helpful in learning to write simple Pascal programs for applications in their subject area. I hope they go on to learn more of the facilities in Pascal; many scientific applications do require data structures other than arrays and types other than integer and real.

Graham H. Kirby is a Lecturer in Compute Studies at the University of Hull. 